\title{
Geriatric Medicine Leadership of Health Care Transformation: To Be or Not To Be?
}

\author{
George A. Heckman, MD, MSc, FRCPC ${ }^{1}$, Frank J. Molnar, MSc, MDCM, FRCPC ${ }^{2}$, \\ Linda Lee, MD, MSISc(FM), CCFP, FCFP ${ }^{3}$ \\ ${ }^{1}$ Research Institute for Aging and School of Public Health and Health Systems, University of Waterloo, Waterloo, ON; \\ ${ }^{2}$ Division of Geriatric Medicine, The University of Ottawa, Ottawa, ON; ${ }^{3}$ The Schlegel-UW Research Institute for \\ Aging and Department of Family Medicine, Kitchener, ON
}

DOI:http://dx.doi.org/10.5770/cgj.16.89

\begin{abstract}
Geriatric Medicine is well-suited to inform and lead healthcare system redesign to address the needs of seniors with complex conditions. We posit that geriatricians must urgently consider how to "brand" Geriatric Medicine in a manner that garners active support from those outside the specialty, including how to adapt practice patterns to better meet the needs of patients and of the health-care system.
\end{abstract}

Key words: geriatric medicine, frailty, health system, primary care, dementia

\section{INTRODUCTION}

Recent data confirms that mounting fiscal pressure on the Canadian health-care system is driven less by ageing and more by the growing chronic disease burden among seniors. ${ }^{(1)}$ Over $75 \%$ of Canadians aged 65 years and over report having at least one chronic condition, and over $40 \%$ have three or more. $(1,2)$ These co-morbidities are often complicated by geriatric conditions, particularly dementia, leading to more hospitalizations, prolonged lengths of stay, higher Alternate Level of Care (ALC) rates, and institutionalization, further straining an already overburdened health-care system. ${ }^{(3)}$ Never before has there been a greater need to redesign the delivery of health care in Canada in order to effectively and efficiently address the needs of seniors with complex conditions.

Geriatric Medicine is particularly well-suited to inform and lead health-care system redesign to meet this challenge. Paradoxically, at this time of greatest need, Geriatric Medicine is severely limited in its ability to assume this leadership role due to a critical shortage of geriatricians in Canada. We posit that geriatricians must urgently consider how to "brand" Geriatric Medicine in a manner that garners active support from those outside the specialty, including how to adapt practice patterns to better meet the needs of patients and of the health-care system.

\section{The Expertise of Geriatric Medicine: Frailty and Multimorbidity}

Geriatricians are experts in frailty and multimorbidity. Frailty is a state of increased vulnerability arising from decreased reserve across multiple physiologic systems. ${ }^{(4)}$ Features include cognitive impairment, weakness, sarcopenia, reduced activity, and falls, which together predispose individuals to disability, health resource utilization, and death. ${ }^{(4)}$ Frailty most often affects seniors in whom it is often associated with multimorbidity. ${ }^{(4)}$ However, most seniors with chronic disease are not frail, and thus a simple co-morbidity count does not fully explain why some seniors do poorly and others age successfully. ${ }^{(4)}$ Other factors must be considered.

The Health and Retirement Study surveyed 11,093 Americans aged 65 years and over residing in a variety of community settings. ${ }^{(5)}$ In the study, geriatric syndromes, such as cognitive impairment, sensory impairment, falls, urinary incontinence, dizziness and weight loss, were not only as common as co-morbidities such as diabetes or heart disease, but were also associated with greater disability. These data show how the concurrent burden of geriatric syndromes and multimorbidity more accurately reflects health status and risk among seniors, consistent with the depiction of frailty resulting from the accumulation of deficits across multiple systems. ${ }^{(4)}$

In order to formulate effective and enduring care plans for frail seniors, additional factors must be addressed, including economic and demographic issues, lifestyle choices, informal support networks, social isolation, and caregiver stress. ${ }^{(6)}$ The assessment of all of these factors and their incorporation into an appropriate plan of care has been organized into a procedure known as the Comprehensive Geriatric Assessment (CGA), a "multidimensional interdisciplinary diagnostic process focused on determining a frail older persons' medical, psychological and functional capacity in order to develop a coordinated and integrated plan for treatment and long-term follow-up". ${ }^{(6)}$ The CGA procedure, when 
applied to frail seniors, results in improved prescribing of medications, better quality of life, fewer falls, reduced rates of hospitalization, reduced institutionalization, and lower mortality rates, at no additional cost and with some studies suggesting a reduction in health-care expenses. ${ }^{(7-11)}$

Geriatricians are certified in Internal Medicine and receive a minimum of two additional years of training as consultants who specialize in CGA, the management of geriatric syndromes, geriatric rehabilitation, geriatric psychiatry, and other subspecialty interests such as osteoporosis and heart failure. ${ }^{(12)}$ Geriatric Medicine is, therefore, a strategically hybridized specialty whose focus is not restricted to a single organ or disease state. Rather it is one that provides integrated training across multiple high-impact specialties to produce highly skilled and flexible physicians optimally prepared to address the needs of complex frail seniors and mitigate their impact on the health-care system. Geriatricians are the decathletes of Internal Medicine.

\section{The Scale of Geriatric Medicine: Inadequate Num- bers for a Critical Mass}

Canada is experiencing a critical shortage of geriatricians. A recent pan-Canadian survey identified 242 certified specialists in Geriatric Medicine, 35\% of whom were aged over 55 years. ${ }^{(13)}$ Compounding this shortage, many practice geriatrics part time due to financial disincentives. ${ }^{(13)}$ Furthermore, while the number of Canadian seniors increased from 2.7 million to 4.8 million between 1986 and 2010, the number of trainees in Geriatric Medicine in Canada remained stagnant at 15 to 25 per year. ${ }^{(13,14)}$ Though the actual number of geriatricians required to meet the needs of the Canadian health-care system is unclear, with previous estimates ranging up to 700 , there is clear agreement that a significant shortage exists. ${ }^{(13)}$

Given that geriatricians have been shown to be among the most satisfied of all medical practitioners, ${ }^{(15)}$ how did this problem of inadequate scale arise? We propose that at least three factors contribute to the inability of Geriatric Medicine to grow rapidly enough to meet escalating societal needs: 1) inadequate exposure of medical trainees to Geriatric Medicine, 2) a need for new strategic directions to match changing societal needs, and 3) the difficulty Geriatric Medicine has in defining itself in terms that those outside the specialty will understand, will feel are meaningful, and will support.

\section{Out of Sight, Out of Mind: Inadequate Exposure of Medical Trainees to Geriatric Medicine}

A 2008-9 survey of 16 Canadian medical schools demonstrated that the mean number of hours of undergraduate geriatric teaching was 82 , a marked contrast to the over 300 hours of pediatrics education. ${ }^{(16,17)}$ This distribution clearly does not reflect societal needs for medical care adapted to an ageing population. Furthermore, while all Psychiatry programs had mandatory rotations in Geriatric Psychiatry, only six Internal Medicine programs had mandatory rotations in Geriatric Medicine. ${ }^{(17)}$ This is concerning, as most trainees choosing to specialize in Geriatric Medicine do so during their Internal Medicine residency. ${ }^{(15)}$ Many geriatricians, including two of the authors of this paper, anecdotally report having been actively discouraged from pursuing a career in Geriatric Medicine during medical school and residency. While some programs are clearly supportive of Geriatric Medicine, there is overall inadequate support in Canadian medical school and Internal Medicine residency programsa factor that is likely contributing to the Geriatric Medicine human resource shortfall.

\section{Right Time, Wrong Place: Are New Strategic Direc- tions Required?}

There is growing concern that not only is this small number of geriatricians spread too thinly, but also that most geriatricians are not involved in activities and sectors where they might make the greatest difference. ${ }^{(18)}$ The National Physician Survey from 2010, sent to all licensed physicians in Canada, revealed that of Geriatric Medicine respondents, the majority were hospital-based, with only $17 \%$ working out of private offices and $12 \%$ working in community settings. ${ }^{(19)}$ This distribution is at odds with recommendations that geriatricians increase their presence in primary care in order to facilitate better community-based collaborative management of frail seniors with complex multimorbidity and reduce the risk of subsequent disability and associated health service utilization. ${ }^{(18)}$ These recommendations were reiterated in the David Walker Report submitted to the Ontario Ministry of Health and Long Term Care in 201, in which it was stated that the "Ministry of Health and Long-Term Care, in consultation with stakeholders, develop a Geriatric Assessment Clinic model that works with and supports family physicians, and takes into consideration the existing primary care models". (20) Emerging evidence strongly suggests that such collaboration can result in improved care, as well as more efficient use of geriatric specialists. ${ }^{(21,22)}$

\section{Garnering External Support: Branding Geriatric Medicine in Meaningful and Understandable Terms} Branding has been described as "not about getting your target market to choose you over the competition, but about getting your prospects to see you as the only one that provides a solution to their problem". ${ }^{(23)}$ A fundamental problem is that Geriatric Medicine is difficult to comprehensively define in terms that others outside the specialty find meaningful, are able to recall, and will support. It is self-evident that a cardiologist deals with cardiac problems. The Royal College of Physicians and Surgeons of Canada (RCPSC) website provides a rather vague definition of Geriatric Medicine as "that branch of medicine which is concerned with the prevention, diagnosis, treatment, and social aspects of illness in older people".(12) This definition is inadequate: most specialties other than paediatrics and obstetrics can lay claim to some 
aspect of "illness of older people"! Geriatric Medicine is far too multi-faceted to be comprehensively captured by a simple definition. Perhaps a better strategy is for the specialty to highlight key core competencies that match the greatest societal needs.

An example of such a core competency is drawn from the area of dementia care. Dementia is clearly the main driver of ALC rates. ${ }^{(3)}$ The House of Commons Standing Committee on Health, in its May 2012 report Chronic Diseases Related to Aging and Health Promotion and Disease Prevention, called dementia the "Godfather of chronic diseases", emphasizing how dementia can destabilize control of concomitant chronic diseases and trigger hospitalizations that ultimately lead to ALC and institutionalization (the "Dementia Domino Effect"). ${ }^{(24)}$ Given recent estimates that as many as three-quarters of a million Canadians have dementia and that over $90 \%$ of community-dwelling seniors with dementia have two or more concomitant chronic diseases, the health-care system is facing the unprecedented challenge of caring for large numbers of persons with concomitant dementia and multiple unstable chronic conditions. $(1,25)$ If plans to address this challenge are not developed soon, ALC rates and wait lists for long-term care homes will continue to grow, leading to further "hospital gridlock", including increased wait times for procedures. ${ }^{(26)}$ Such planning must encompass "full spectrum dementia care" that involves all specialties that diagnose and care for persons with dementia (e.g., Geriatric Medicine, Care of the Elderly, Family Medicine, Neurology, Psychiatry, Geriatric Psychiatry) working in close collaboration. In particular, the most medically complex patients will require access to specialists not only trained in dementia care, but also qualified in Internal Medicine and in CGA. The only specialty that can claim such expertise is Geriatric Medicine. This expertise is a clear example of a high-impact core competency that Geriatric Medicine must highlight in order to brand itself in a manner that will attract the attention, acknowledgement, and support of other specialties and decision makers.

\section{The Challenge for Geriatric Medicine: a Call to Arms}

Defining the geriatric brand, by achieving consensus on a standard definition and identifying the core competencies of Geriatric Medicine and marketing these to external stakeholders, requires urgent attention. Unless we rapidly increase the number of geriatricians and direct new graduates to strategic areas where they can have the greatest impact, the health-care system redesign required to meet the growing needs of frail seniors with multiple co-morbidities will proceed only with great difficulty, if at all. If Geriatric Medicine is to take on a leadership role in health-care transformation, then immediate action is required at several levels:

1. The Canadian Geriatrics Society (CGS) should ignite a national debate, not just amongst geriatricians but also with external stakeholders, to achieve consensus on a definition of the specialty and affirm its core competencies.

2. The Canadian Medical Association (CMA), the RCPSC, and provincial and federal ministries of health must recognize that the core competencies of Geriatric Medicine are key to addressing the escalating pressure on the healthcare system from seniors with complex co-morbidities often complicated by cognitive impairment; effectively implemented, the CGA can improve patient outcomes, reduce resource utilization, and potentially save money.

3. The above stakeholders must acknowledge that while several specialties face human resource challenges, the situation in Geriatric Medicine is most precarious. It is deeply paradoxical that while trainees in some specialties are facing unemployment, Geriatric Medicine, one of the most satisfying of medical specialties, ${ }^{(15)}$ cannot attract enough recruits to meet the escalating needs of an ageing population. ${ }^{(27)}$ Medical schools, and particularly Internal Medicine programs, must allocate more time to training in Geriatric Medicine. Physician resource planning in Canada is flawed and in urgent need of review.

4. Primary care must become more aware of the value of involving geriatricians in a more proactive manner, rather than waiting until a health crisis is imminent or has occurred. The CGS and the Canadian College of Family Physicians must collaborate closely to develop optimal models of shared community care for frail seniors.

5. Seniors' organizations must actively engage in this issue if they wish for their members, friends, and families to have the best care possible when their health-care needs become complicated by multimorbidity and cognitive impairment. Without the voice of this key and politically influential sector of society, meaningful progress may not occur.

A call to arms has been sounded. Leadership, vision, and action are required now if we are to effect successful healthcare redesign to address the escalating care needs of seniors with complex multimorbidities, and avert further increases to ALC rates, longer wait times, and more hospital gridlock. Geriatricians can play leadership roles in providing expertise for system redesign and support strategic health-care sectors such as primary care. Now is the time for all health-care stakeholders to understand and affirm the importance and relevance of Geriatric Medicine.

\section{ACKNOWLEDGEMENTS}

The authors wish to thank Ms. Michelle Klosch, Assistant Head of English at Huron Heights Secondary School, for suggesting the title of this article. Essentially, Shakespeare's Hamlet is seen as an introspective protagonist who, despite struggling with existential questions, fails to take action, leading to unfortunate outcomes not only for himself, but for those around him. 


\section{CONFLICT OF INTEREST DISCLOSURES}

The authors declare that no conflicts of interest exist.

\section{REFERENCES}

1. Canadian Institute for Health Information. Seniros and the health care system: what is the impact of multiple chronic conditions? Ottawa: CIHI;2011. Available from: https://secure. cihi.ca/estore/productFamily.htm?locale=en\&pf $=$ PFC1575 (Accessed 2012 August 22).

2. Rapoport J, Jacobs P, Bell NR, et al. Refining the measurement of the economic burden of chronic diseases in Canada. Chronic Dis Canada. 2004;25(1):13-21.

3. Canadian Institute for Health Information. Alternate level of care in Canada Ottawa: CIHI; 2009. Available at: https://secure.cihi. ca/estore/productFamily.htm?pf=PFC1097\&lang=fr\&media $=0$ (Accessed 2012 December 18).

4. Bergman H, Ferrucci L, Guralnikm J, et al. Frailty: an emerging research and clinical paradigm - issues and controversies. J Gerontol A Biol Sci Med Sci. 2007;62(7):731-37.

5. Cigolle CT, Langa KM, Kabeto MU, et al. Geriatric conditions and disability: the Health and Retirement Study. Ann Intern Med. 2007;147(3):156-64.

6. Abellan van Kan G, Rolland Y, Houles M, et al. The assessment of frailty in older adults. Clin Geriatr Med. 2010 May;26(2):275-86.

7. Schmader KE, Hanlon JT, Pieper CF, et al. Effects of geriatric evaluation and management on adverse drug reactions and suboptimal prescribing in the frail elderly. Am J Med. 2004;116(6):394-401.

8. Beswick AD, Rees K, Dieppe P, et al. Complex interventions to improve physical function and maintain independent living in elderly people: a systematic review and meta-analysis. Lancet. 2008;371(9614):725-35.

9. Day P, Rasmussen P. What is the evidence for the effectiveness of specialist geriatric services in acute, post-acute and sub-acute settings? A critical appraisal of the literature. New Zealand Health Technology (NZHTA) Report. 2004;7(3):1-169.

10. Challis D, Clarkson P, Williamson J, et al. The value of specialist clinical assessment of older people prior to entry to care homes. Age Ageing. 2004;33(1):25-34.

11. Ellis G, Whitehead MA, Robinson D, et al. Comprehensive geriatric assessment for older adults admitted to hospital: metaanalysis of randomised controlled trials. BMJ. 2011;343:d6553

12. Royal College of Physicians and Surgeons: Specialty profiles. Available at: http://www.royalcollege.ca/portal/page/portal/rc/ public (Accessed 2012 December 18).

13. Hogan DB, Borrie M, Basran JFS, et al. Specialist physicians in geriatrics. Report of the Canadian Geriatrics Society Physician Resource Work Group. Can Geriatr J. 2012;15(3):68-79.

14. Canadian Institute for Health Information. Health care in Canada, 2011 — a focus on seniors and aging. Ottawa: CIHI; 2011. Available from: https://secure.cihi.ca/estore/productFamily. htm?locale=en\&pf=PFC1677 (Accessed 2012 December 18).
15. National Physician Survey 2004. Available from: http://national physiciansurvey.ca/surveys/2004-survey/ (Accessed 2012 December 18)

16. Gordon JE. Updated survey of the geriatric content of Canadian undergraduate and postgraduate medical curricula. Can Geriatr J. 2011;14(2):34-39.

17. Gordon J, Hogan D. Survey of the geriatric content of Canadian undergraduate and postgraduate medical curricula. Can Geriatr J. 2006;9(Suppl 1):S6-S11.

18. Patterson $\mathrm{C}$, Hogan DB, Bergman $\mathrm{H}$. The choices facing geriatrics. Can Geriatr J. 2012;15(2):24-27.

19. National Physician Survey 2010. Available from: http://national physiciansurvey.ca/surveys/2010-survey/ (Accessed 2012 December 18)

20. Walker D. Caring for our aging population and addressing alternate level of care: report submitted to the Minister of Health and Long Term Care. 2011. Available at: http://www.homecareontario. $\mathrm{ca} /$ documanager/files/news/report--walker_2011--ontario.pdf (Accessed 2012 December 18).

21. Lee L, Hillier LM, Stolee P, et al. Enhancing dementia care: a primary care-based memory clinic. J Am Geriatr Soc. 2010;58(11):2197-204.

22. D'Arcy LP, Stearns SC, Domino ME, et al. Is geriatric care associated with less emergency department use? J Am Geriatr Soc. 2013;61(1):4-11.

23. Lake L. What is branding and how important is it to your marketing strategy? [Internet] Marketing.about.com. Available at: http://marketing.about.com/cs/brandmktg/a/whatisbranding. htm (Accessed 2012 December 18).

24. Smith J. Chronic diseases related to aging and health promotion and disease prevention. Report for the Standing Committee on Health. May 2012. Available at: http:/www.parl.gc.ca/content/ hoc/Committee/411/HESA/Reports/RP5600467/hesarp08/ hesarp08-e.pdf (Accessed 2012 December 18).

25. Institute for Clinical Evaluative Sciences. Health system use by frail Ontario seniors. An in-depth examination of four vulnerable cholorts. Toronto: Institute for Clinical Evaluative Sciences; 2011. Available at: http://www.ices.on.ca/file/ ICES_AgingReport_2011.pdf (Accessed 2012 December 18).

26. Wait Times Alliance of Canada. Shedding light on Canadians' total wait for care: report card on wait times in Canada. Ottawa: Wait Times Alliance of Canada; 2012. http:/www.waittimeal liance.ca/media/2012reportcard/WTA2012-reportcard_e.pdf (Accessed 2012 December 30).

27. Taggart K. New orthopedic surgeons facing spectre of unemployment, meeting told [Internet]. CMA News July 242012. Available at: http://www.cma.ca/new-orthopedic-surgeonsunemployment (Accessed 2012 December 19).

Correspondence to: George A. Heckman, MD, MSc, FRCPC, Research Institute for Aging and School of Public Health and Health Systems, University of Waterloo, BMH 3734, 200 University Avenue West, Waterloo, ON, N2L 3G1, Canada E-mail: ggheckma@uwaterloo.ca 